\title{
Lower values of handgrip strength and adductor pollicis muscle thickness are associated with hepatic encephalopathy manifestations in cirrhotic patients
}

\author{
L. Augusti ${ }^{1}$ L. C. Franzoni $^{1}$ - L. A. A. Santos ${ }^{1}$ - T. B. Lima ${ }^{1}$ - M. V. Ietsugu ${ }^{1}$ • \\ K. H. Koga ${ }^{2}$ - S. M. Moriguchi ${ }^{2}$ L. E. Betting ${ }^{3}$ C. A. Caramori ${ }^{1}$ - G. F. Silva ${ }^{1}$ • \\ F. G. Romeiro ${ }^{1}$
}

Received: 12 February 2016 / Accepted: 22 April 2016/Published online: 30 April 2016

(C) Springer Science+Business Media New York 2016

\begin{abstract}
Hepatic encephalopathy (HE) is a late complication of liver cirrhosis and is clearly associated with poor outcomes. Chronic liver insufficiency leads to progressive muscle wasting, impairing ammonia metabolism and thus increasing the risk for HE. Given the association between lean mass and adductor pollicis muscle thickness (APMT), it has been used to predict outcome and complications in many conditions, but not yet in cirrhotic patients. Therefore, this article aimed to study the association between HE manifestations and measures related to muscle mass and strength. This cross-sectional study included 54 cirrhotic outpatients with HE varying from subclinical to grade II according to the West-Haven criteria, who were submitted to neuropsychometric tests, electroencephalogram, brain Single Photon Emission Computed Tomography (SPECT), anthropometric measurements, handgrip strength (HGS) and dual energy X-ray absorptiometry exam (DXA). Multiple logistic regression analysis was performed to investigate the association between body composition measures and HE grade. Analysis of the area under the receiver operator characteristic (AUROC) curve revealed the values related to neurological manifestations (HE grades I and II). Reductions
\end{abstract}

F. G. Romeiro

fgromeiro@fmb.unesp.br

1 Department of Internal Medicine - Botucatu Medical School, UNESP- Univ Estadual Paulista, Av. Prof. Mário Rubens Guimarães Montenegro, s/n., Distrito de Rubião Jr., Botucatu, São Paulo 18618 687, Brazil

2 Department of Tropical Diseases and Imaging Diagnosis, Botucatu Medical School, UNESP - Univ Estadual Paulista, Botucatu, Brazil

3 Department of Neurology, Psychology and Psychiatry, Botucatu Medical School, UNESP - Univ Estadual Paulista, Botucatu, Brazil in APMT and HGS were associated with higher HE grades, suggesting a big impact caused by the loss of muscle mass and function on HE severity. The link between HE manifestations and anthropometric measures, namely APMT and HGS, point to a significant relation concerning skeletal muscles and the neurological impairment in this population.

Keywords Hepatic encephalopathy $\cdot$ Liver cirrhosis . Anthropometry $\cdot$ Muscle strength

\section{Introduction}

Hepatic encephalopathy (HE) is one of the worst consequences of chronic liver failure, affecting both patient and caregiver quality of life (Bajaj et al. 2011; Bianchi et al. 2012; Vilstrup et al. 2014a, b). It is also linked to high mortality rates among patients with liver cirrhosis (Stewart et al. 2007; Romero-Gómez et al. 2015). In HE physiopathology, inflammation and ammonia homeostasis involve multiple organ systems, namely the gut, the kidneys and the muscles (Tapper et al. 2015).

Skeletal muscles can reduce levels of ammonia by converting it to glutamine via glutamine synthetase, thus decreasing ammonia content and avoiding HE manifestations (Butterworth 2002; Vilstrup et al. 2014a, b; Tapper et al. 2015). Prognosis of cirrhotic patients is noticeably affected by muscle loss, while patients with sarcopenia or malnutrition have an increased risk of HE (Romeiro and Augusti 2015; Kalaitzakis et al. 2007; Huisman et al. 2011; Montano-Loza et al. 2012; Merli et al. 2013). Therefore, nutritional assessment of these patients is essential, with special attention on their muscle mass, in order to prevent or treat possible nutritional disorders. 
However, the assessment of body composition in cirrhotic patients is a challenging task. They often present fluid retention, even before the clinical detection of ascites or edema (McCullough et al. 1991). Cirrhosis leads to a high catabolism of body fat and lean mass, which after a short overnight fasting period is comparable to that of healthy individuals after 23 days of starvation (Cheung et al. 2012). Loss of muscle mass is predominant in these patients, suggesting that sarcopenia is the best definition for their nutritional disorder (Periyalwar and Dasarathy 2012). As muscle wasting is not strictly accompanied by body fat depletion, they can also present overweight or obesity, so in this last condition they are classified as sarcopenic-obese (Berzigotti et al. 2011; Jeejeebhoy 2012).

The diagnosis of sarcopenia and sarcopenic obesity can be obtained from the dual energy X-ray absorptiometry exam (DXA). This method allows the calculation of the appendicular muscle mass index (AMMI), which has already been applied to cirrhotic patients for providing an accurate measure of lean mass, less influenced by hydration status and overweight (Hayashi et al. 2013). Individuals with AMMI lower than two standard deviations below the gender-specific reference value for healthy adults $\left(<7.26 \mathrm{~kg} / \mathrm{m}^{2}\right.$ for men and $<5.45 \mathrm{~kg} / \mathrm{m}^{2}$ for women) are diagnosed as sarcopenic; sarcopenic obesity occurs when sarcopenia is associated with fat mass percentages greater than $27 \%$ for men and above $38 \%$ for women (Baumgartner et al. 1998; Baumgartner 2000; Waters et al. 2008; Prado et al. 2012). In spite of the advantages of DXA for evaluating body composition changes, it is not recommended as a follow-up method because of the ionizing radiation involved. Another limitation is that DXA is not easily applicable to HE patients. Therefore, bedside measures constitute the most frequently applied techniques for evaluating these patients in routine assessments.

Despite some discussion related to accuracy and reproducibility, anthropometric measures are widely useful and recommended for cirrhotic patients (Plauth et al. 2006; Bémeur et al. 2010; Chadalavada et al. 2010). These methods are radiationfree, low-cost and easy-to-perform techniques that can be applied over time in HE patients. Of note, some bedside techniques such as handgrip strength (HGS) and mid-arm muscle circumference (MAMC) are good indicators of which patients are at risk for HE, but few studies evaluated these associations between anthropometric indicators and HE (Álvares-da-Silva 2005; Merli et al. 2013). Therefore, this study aimed to analyze the relations between HGS, body composition measures and HE severity in cirrhotic patients.

\section{Methods}

It was a cross-sectional study in which cirrhotic patients with HE and aged more than 18 years were selected to participate. The study was approved by the local Ethics Committee (protocol 4334-2012) and carried out according to the Declaration of Helsinki and its revisions. Informed consent was obtained from all individual participants included in the study. The diagnosis of cirrhosis was made by liver biopsy or by associating clinical, laboratorial and imaging exams (radiological and/or endoscopic findings compatible with cirrhosis and portal hypertension). Psychometric tests (number connection test), electroencephalogram (EEG) and brain Single Photon Emission Computed Tomography (SPECT) were performed to assess psychometric performance, cortical activity and cerebral perfusion, respectively.

HE staging was based on the West Haven criteria described by Blei et al., further endorsed by Amodio et al. and improved by the recent Guidelines (Blei 2000; Blei and Córdoba 2001; Amodio et al. 2004; Vilstrup et al. 2014a, b). Grade 0 included individuals without neurological manifestations, whose diagnosis was made only from the results of the aforementioned exams. Grades I and II were established according to the clinical manifestations, which were documented by two skilled doctors (FGR and LCF) on specific forms, detailing all symptoms and signals presented by the patients according to the current Guidelines (Vilstrup et al. 2014a, b). Patients with excessive somnolence, hepatic coma or other serious neuropsychiatric diseases were excluded. The dependent variable was the HE grade while the independent variables were age, handgrip strength, Model for end stage liver disease (MELD) score and body composition measures from anthropometry and DXA.

\section{Study protocol}

The subjects were submitted to EEG, brain SPECT, clinical exams, laboratory tests and body composition evaluation, which was composed of anthropometric measures, handgrip strength test and DXA. The clinical exam was focused on ascites and HE grading. Laboratory tests included albumin, ammonia, creatinine, international normalized ratio and total bilirubin.

Interictal EEG was performed with a 32-channel recorder (Nihon Kohden, Tokyo, Japan). Electrodes were positioned according to the 10-20 international system of electrode placement. All records were performed with 20 min long, with photic stimulation and hyperventilation, and were qualitatively analyzed by one neurologist who was unaware of the clinical data. The exams were divided in normal or abnormal according to the presence of electroencephalographic signs of encephalopathy based on the background activity (Amodio et al. 1999).

Brain SPECT was performed with the patient in supine position and free from audiovisual stimulus for at least 20 min before the acquisitions. Alcohol, caffeine and stimulants were prohibited since the day before the exam. One hour before the acquisition, the subjects received $1110 \mathrm{MBq}$ 
$(30 \mathrm{mCi})$ of $99 \mathrm{mTc}-$ bicisate ethyl cysteinate dimer (ECD) through a peripheral vein. Images were acquired by a dualheaded gamma camera (Millennium MG system, General Electric, United Kingdom). The photopeak data were obtained with a $20 \%$ energy centered at $140 \mathrm{keV}$ using low energy and high-resolution (LEHR) collimators. The data were acquired in a $128 \times 128$ matrix over $360^{\circ}$ rotation performing 65 frames of $25 \mathrm{~s}$ duration. Filtered backprojection reconstruction (Butterworth filter 5 , critical frequency of 0.5 cycles $/ \mathrm{min}$ ) was applied as recommended (Morano and Seibyl 2003; Sunil et al. 2012). A specialist who was unaware of the clinical data used the software Statistical Parametric Mapping (SPM) (Wellcome Trust Centre for Neuroimaging, London) version 8 for the analysis. The exams were divided in normal or abnormal according to the presence of regions of abnormal cerebral perfusion (Sunil et al. 2012).

Anthropometric measurements collected were weight, height, arm circumference (AC), triceps skinfold (TSF) and adductor pollicis muscle thickness (APMT). Weight and height were measured on a digital scale platform with stadiometer (Filizola ${ }^{\circledR}$ ID 1500), in which the weight values obtained were adjusted according to the presence of edema and/or ascites as previously recommended (James 1989). The body mass index (BMI) was obtained by calculating the dry weight divided by the square of the height.

After finding the midpoint between the acromion and the olecranon, AC and TSF were measured as previously stated (Callaway et al. 1988; Harrison et al. 1988). To measure TSF, the fold of fat was pinched with the caliper (Lange ${ }^{\circledR}$ ) and the mean value of three measures was registered. APMT was measured with the arm bent at $90^{\circ}$ and with the dominant hand relaxed on the knee, using the same caliper and pinching the adductor muscle between the thumb and the forefinger, registering the mean value of 3 measurements, as recommended (Lameu et al. 2004). The mid-arm muscle circumference (MAMC) and the correct mid-arm muscle area (CAMA) was then obtained using the following formulas: MAMC $(\mathrm{cm})=A C-\pi \times(T S F \div 10)$ and CAMA $\left(\mathrm{cm}^{2}\right)=$ $\left[(A C-\pi \times T S F \div 10)^{2} / 4 \pi\right]-6.5$ for women and CAMA $\left(\mathrm{cm}^{2}\right)=\left[(A C-\pi \times T S F \div 10)^{2} / 4 \pi\right]-10$ for men (Blackburn and Harvey 1982).

Handgrip strength (HGS) was registered by a dynamometer (Saehan grip - Saehan Corporation, South Korea) encouraging the patients to maximize their effort during each strength test, which were measured from the non-dominant hand with the patient seated and the forearm flexed from the elbow at a $90^{\circ}$ angle. Three attempts were required, with $30 \mathrm{~s}$ of resting between each one, then registering the highest value obtained (Álvares-da-Silva 2005).

The DXA (Discovery QDR - Hologic, Inc.) was performed to obtain the appendicular muscle mass index (AMMI), calculated by dividing the sum of appendicular muscle mass of the four members, free of fat and bone, by the square of the height (Baumgartner et al. 1998).

\section{Statistical analysis}

Descriptive analysis was based on mean and standard deviation (SD) for parametric data and on median and interquartile ranges for non-parametric data. The sample was divided into three groups based on HE grades, and multiple logistic regression analysis was performed to analyze the association between anthropometric measurements and HE severity. Then, the cutoff points of the significant variables were calculated by the area under receiver operator characteristic (AUROC) curve analysis to find the values associated with neurological manifestations (HE grades I and II). The statistical significance level was defined as $p<0.05$. The software SAS (Statistical Analysis System) for Windows, v.9.3 was used to make the analysis.

\section{Results}

Sixty-three subjects were recruited from hepatology outpatient appointments. Five of them died and four were excluded for not completing the nutritional assessment. Thus, the sample consisted of 54 individuals ( 30 men and 24 women) aged $56.9 \pm 11$ years-old with a median MELD score of 13 points. Most of the subjects had Child-Pugh B cirrhosis and HE grade I. All the patients had altered psychometric tests, and the median value obtained from the number connection test was 85 points $(63.25-193.25)$. One subject died before undergoing the EEG and another one did not return to accomplish the EEG and the SPECT exams. All the other patients completed the study protocol. The subjects' characteristics are summarized in Table 1. The main neurological manifestations presented by patients with HE grades I and II were psychomotor slowing ( 25 subjects), asterixis ( 23 subjects), shortened attention span (21 subjects), disturbances of the sleepwake cycle (17 subjects), slowness of speech (14 subjects) and mild personality changes (9 subjects).

The results of anthropometric measurements, handgrip strength and AMMI are shown in Table 2. Based on the AMMI values, $17 \%$ of the subjects had sarcopenia (5 men and 4 women).

In the logistic regression model, APMT and HGS were the only anthropometrics measurements related to changes in HE grade. Reductions of $1 \mathrm{~mm}$ in APMT and $1 \mathrm{~kg}$ in HGS were associated with odds of $30.7 \%$ and $12.2 \%$ of increasing HE grade, respectively (Table 3). The odds of change between HE Grades 0 and I were the same for changing between the grades I and II.

Given that APMT and HGS were related to alterations in HE grade, the discriminative power of the model was 
Table 1 Main characteristics of the sample $(n=54)$

\begin{tabular}{|c|c|c|c|}
\hline Variables & Categories & Subjects & Percentages \\
\hline \multirow[t]{2}{*}{ Sex } & Men & 30 & 55.6 \\
\hline & Women & 24 & 44.4 \\
\hline \multirow[t]{3}{*}{ Age group (years) } & $20-40$ & 5 & 9.2 \\
\hline & $40-60$ & 28 & 51.9 \\
\hline & $60-80$ & 21 & 38.9 \\
\hline \multirow[t]{3}{*}{ Cirrhosis etiology } & Alcohol & 22 & 37.0 \\
\hline & Viral Hepatitis & 18 & 33.4 \\
\hline & Other causes $^{\mathrm{a}}$ & 14 & 29.6 \\
\hline \multirow[t]{3}{*}{ Child-Pugh classification } & A & 12 & 22.2 \\
\hline & $\mathrm{B}$ & 36 & 66.7 \\
\hline & $\mathrm{C}$ & 6 & 11.1 \\
\hline \multirow[t]{3}{*}{ HE Grades } & 0 & 20 & 37 \\
\hline & I & 31 & 57.4 \\
\hline & II & 3 & 5.6 \\
\hline \multirow[t]{2}{*}{ EEG results $(n=52)$} & Normal & 21 & 40 \\
\hline & Altered & 31 & 60 \\
\hline \multirow[t]{2}{*}{ Brain SPECT results $(n=53)$} & Normal & 25 & 47 \\
\hline & Altered & 28 & 53 \\
\hline
\end{tabular}

a autoimmune hepatitis $(n=5)$, non-alcoholic fatty liver disease $(n=3)$ and cryptogenic cirrhosis $(n=6)$. $E E G$ electroencephalogram, $H E$ hepatic encephalopathy, SPECT Single Photon Emission Computed Tomography. HE grading was performed according to the West Haven criteria

evaluated by the ROC curve analysis to determine their best cutoff values as markers of neurological manifestations (grades I and II), as shown in Fig. 1. APMT lower than $6.5 \mathrm{~mm}$ and HGS lower than $20.5 \mathrm{~kg}$ were associated with findings of HE grades I and II, as shown in Table 4.

Table 2 Anthropometric measures, handgrip strength and appendicular muscle mass index from the dual energy X-ray absorptiometry exam

\begin{tabular}{ll}
\hline Variable & Values \\
\hline AC $(\mathrm{cm})$ & $28.6 \pm 4.8^{\mathrm{a}}$ \\
AMMI $\left(\mathrm{kg} / \mathrm{m}^{2}\right)$ & $7.61(1.38)^{\mathrm{a}}$ \\
APMT $(\mathrm{mm})$ & $7.1(5-8)^{\mathrm{b}}$ \\
BMI $\left(\mathrm{kg} / \mathrm{m}^{2}\right)$ & $26.8 \pm 4.7^{\mathrm{a}}$ \\
CAMA $\left(\mathrm{cm}^{2}\right)$ & $36.6 \pm 13.2^{\mathrm{a}}$ \\
HGS $(\mathrm{kg})$ & $20.3(16-25.7)^{\mathrm{b}}$ \\
MAMC $(\mathrm{cm})$ & $23.5 \pm 3.7^{\mathrm{a}}$ \\
TSF $(\mathrm{mm})$ & $16.1 \pm 6.6^{\mathrm{a}}$ \\
\hline
\end{tabular}

$A C$ arm circumference, $A M M I$ appendicular muscle mass index, $A P M T$ adductor pollicis muscle thickness, $B M I$ body mass index, $C A M A$ corrected arm muscle area, $H G S$ handgrip strength, $M A M C$ mid-arm muscle circumference, $T S F$ triceps skin fold, $\mathrm{kg}$ kilogram, $\mathrm{kg} / \mathrm{m}^{2}$ kilograms per square meter, $\mathrm{cm}$ centimeter, $\mathrm{mm}$ millimeter

${ }^{\mathrm{a}}$ mean \pm standard deviation

${ }^{\mathrm{b}}$ median (1st quartile -3 rd quartile)
Table 3 Multiple logistic regression model for hepatic encephalopathy grade

\begin{tabular}{llll}
\hline Variable & OR & $95 \% \mathrm{CI}$ & $p$ \\
\hline AMMI & 1.000 & $1.00-1.00$ & 0.1486 \\
APMT & 1.307 & $1.05-1.63$ & $0.0177^{*}$ \\
BMI & 1.049 & $0.86-1.27$ & 0.6301 \\
HGS & 1.122 & $1.02-1.25$ & $0.0230^{*}$ \\
MAMC & 0.990 & $0.92-1.07$ & 0.7857 \\
\hline
\end{tabular}

$A M M I$ appendicular muscle mass index, $A P M T$ adductor pollicis muscle thickness, $B M I$ body mass index, $C I$ Confidence Interval, $H G S$ handgrip strength, $M A M C$ mid-arm muscle circumference, $O R$ odds ratio. The analysis was adjusted for age and MELD (model for end stage liver disease). Arm circumference (AC), triceps skin fold (TSF) and corrected arm muscle area (CAMA) could not fit in this model

*significant values

\section{Discussion}

The role of skeletal muscles and the central nervous system in ammonia metabolism is strikingly increased in chronic liver failure, making HE a special cirrhosis complication because it requires muscle mass maintenance to avoid neurological damage. However, cirrhosis is a catabolic disease that provokes substantial changes in body composition, leading to progressive muscle wasting. In this study, according to the multiple logistic regression analysis, a $1 \mathrm{~mm}$ reduction in APMT was associated with a $30 \%$ risk of HE grade increasing, whereas a $1 \mathrm{~kg}$ loss in HGS would increase this risk by $12 \%$. These results show that cirrhotic patients with $\mathrm{HE}$ can present muscle depletion and impaired muscle strength, herein represented by APMT and HGS, respectively.

APMT has a strong correlation with lean mass assessed by DXA (Bielemann et al. 2015). It is little influenced by water retention or fat mass, is easy to perform and does not require too much patient collaboration or expensive equipment. This measure has been applied in different groups of patients as a predictor of a worse prognosis (de Oliveira et al. 2012; Caporossi et al. 2012; Poziomyck et al. 2012). However, it can vary according to body composition, gender or even the manual tasks performed over time, so it does not seem to be a suitable method for evaluating dissimilar patients. For instance, APMT was not able to discriminate mortality or length of stay in an intensive care unit (ICU), where the standard deviation values were high and inaccuracy at predicting outcomes was also observed when the authors analyzed MAMC (Leong Shu-Fen et al. 2015). Of note, MAMC is a wellknown predictor of outcome in cirrhotic populations and of HE occurrence (Merli et al. 2013). In contrast to this finding in ICU patients, another study revealed that in chronic kidney disease (CKD) patients, a specific population under risk of malnutrition, APMT values $\leq 10.6 \mathrm{~mm}$ were associated with a hospitalization risk more than 3 times higher (de Oliveira et al. 
Fig. 1 Area under the receiver operating characteristic (AUROC) curves for adductor pollicis muscle thickness (APMT) and handgrip strength (HGS) values as markers of hepatic encephalopathy manifestations (grades I and II)

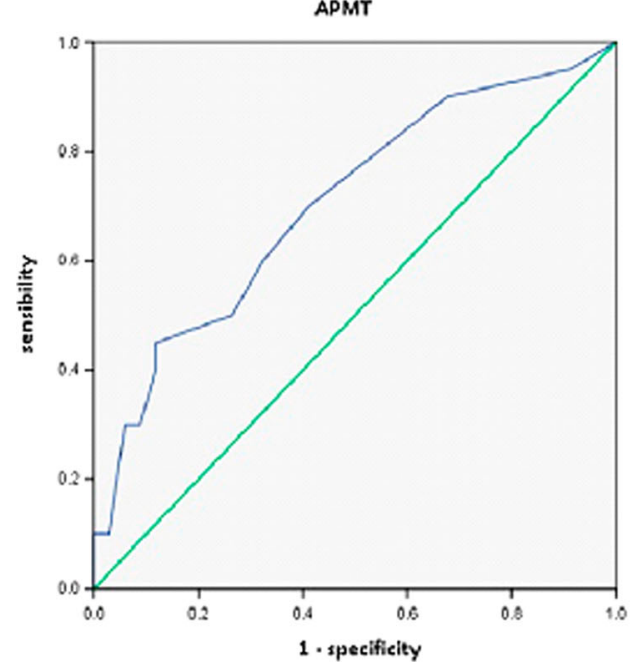

HGS

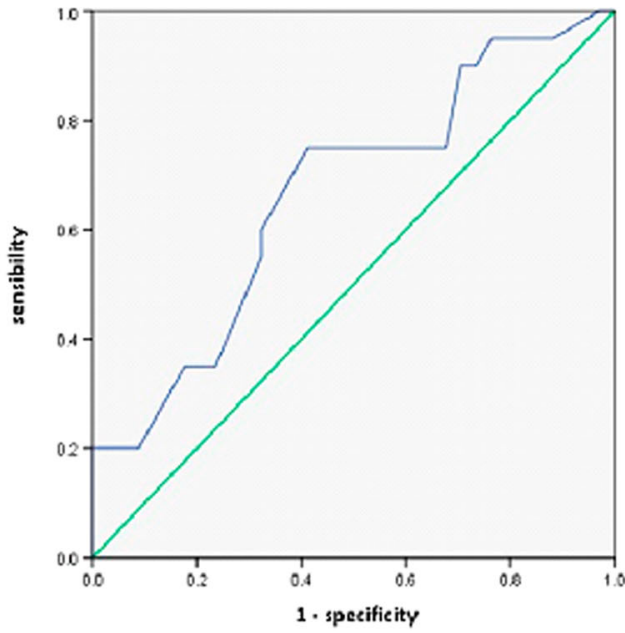

2012). Therefore, similar results could be expected in cirrhotic patients, especially in those with $\mathrm{HE}$, because they are often stricken by nutritional problems such as muscle wasting.

Men and women are affected differentially not only by liver cirrhosis but also by the pattern of muscle loss in different muscles. In cirrhotic patients, MAMC values are more affected in men, whereas the fat loss indicated by TSF is more significant in women (Carvalho and Parise 2006). DXA results confirmed that women present sharper diminution of fat deposits but greater preservation of lean mass, whereas the loss of lean mass is more evident in men (Riggio et al. 1997). In relation to muscle groups, a study using bioelectrical impedance analysis suggested that cirrhosis affects primarily the arms, because arm skeletal muscle mass index was more depleted than leg and appendicular indexes and was also associated with high mortality (Iwasa et al. 2014). The conclusions reported by these authors corroborate the current study, which was focused on measures related to arm muscles and had similar proportions of men and women, some of whom presented with signs of muscle wasting according to the AMMI values.

The findings observed herein point to a significant association between muscle mass measures and HE manifestations. APMT and HGS reductions were relevant despite the differences in body composition within the sample evaluated,

Table 4 Cutoff values for adductor pollicis muscle thickness and handgrip strength as markers of HE Grades I and II

\begin{tabular}{llll}
\hline Variable & Cutoff value & AUROC (CI 95 \%) & $p$ \\
\hline APMT (mm) & 6.5 & $0.70(0.55-0.85)$ & $0.013^{*}$ \\
HGS $(\mathrm{kg})$ & 20.5 & $0.66(0.51-0.81)$ & $0.044^{*}$ \\
\hline
\end{tabular}

$A P M T$ adductor pollicis muscle thickness, $A U R O C$ area under the receiver operating characteristic curve, $H G S$ handgrip strength

*significant values which had subjects with advanced liver disease, different HE grades and dissimilar patterns of muscle wasting. Despite the link between skeletal muscle loss and neurological findings found in this study, a cause effect relationship has still to be proven by longitudinal studies.

Considering that both APMT and HGS are related to arm muscles and are affected in cirrhotic patients, they could be useful markers of mental impairment in this population. As already stated, CKD patients with APMT values $\leq 10.6$ suffered more hospitalizations, while all the subjects evaluated in this study were outpatients and had APMT values varying from 3 to $25 \mathrm{~mm}$, of which those with the lower values had a worse mental status. Unfortunately, we have not found another study with APMT measures from cirrhotic patients that would enable comparisons with the values observed.

HGS is a marker of muscle function widely used to identify malnutrition; some studies have already indicated its relationship with prognosis in cirrhosis (Figueiredo et al. 2000; Álvares-da-Silva 2005; Peng et al. 2007; Huisman et al. 2011). A prior study revealed that cirrhotic patients with overt HE had lower values of HGS and MAMC. The authors concluded that patients had muscle depletion when MAMC values were $<5$ th percentile according to standard values for the general population matched for age and sex. The same was applied for the diagnosis of decreased muscle strength using HGS values, showing that the prevalence of overt HE was much higher in those with these two findings (Merli et al. 2013). In the present study, the AUROC curve analysis showed that HGS values lower than $20.5 \mathrm{~kg}$ were associated with the risk of increasing HE severity from subclinical to grades I or II, which are clearly related to poor prognosis.

By combining the results obtained in this study with those previously reported, it can be inferred that muscle mass and strength have a big impact on cirrhotic patients. The further steps are to discover how to preserve them in this population and whether it would allow decreasing the progression of 
cirrhosis complications such as HE. Despite the lack of studies on APMT in this population, APMT, HGS and MAMC have been suggested as good measures to evaluate muscle mass in cirrhotic patients with HE (Romeiro and Augusti 2015; Merli et al. 2013). According to the findings obtained in this study, the association of APMT and HGS with HE manifestations favors the use of these measures for nutritional assessment of cirrhotic patients.

In conclusion, the results found showed that APMT and HGS are associated with HE neurological manifestations in cirrhotic outpatients stricken by different grades of HE. As these bedside techniques are low-cost, easy-to-perform and radiation-free methods, it is suggested that they should be widely applied to these patients to show which of them must be carefully evaluated to avoid skeletal muscle loss, which is a marker of cirrhosis complications.

Acknowledgments The authors are grateful to EAP (Escritório de Apoio à Pesquisa - Botucatu Medical School/UNESP) and UPECLIN (Unidade de Pesquisa Clínica - UNESP) for their support. They also wish to thank the financial support received from Coordenação de Aperfeiçoamento de Pessoal de Nível Superior (Capes), Pró-Reitoria de Pesquisa (PROPe/UNESP) and São Paulo Research Foundation (FAPESP) grants No. 2013/15121-8 and 2013/11761-2.

\section{Compliance with ethical standards}

Conflict of interest The authors declare that they have no conflict of interest.

Ethical approval All procedures performed involving human participants were in accordance with the ethical standards of the institutional and/or national research committee and with the 1964 Helsinki declaration and its later amendments or comparable ethical standards.

\section{References}

Álvares-da-Silva MR (2005) Reverbel da Silveira T. Comparison between handgrip strength, subjective global assessment, and prognostic nutritional index in assessing malnutrition and predicting clinical outcome in cirrhotic outpatients. Nutrition 21(2):113-117

Amodio P, Marchetti P, Del Piccolo F, de Tourtchaninoff M, Varghese P, Zuliani C, Campo G, Gatta A, Guérit JM (1999) Spectral versus visual EEG analysis in mild hepatic encephalopathy. Clin Neurophysiol 110(8):1334-1344

Amodio P, Montagnese S, Gatta A, Morgan MY (2004) Characteristics of minimal hepatic encephalopathy. Metab Brain Dis 19(3-4):253-267

Bajaj JS, Wade JB, Gibson DP, Heuman DM, Thacker LR, Sterling RK, Stravitz RT, Luketic V, FucHGS M, White MB, Bell DE, Gilles H, Morton K, Noble N, Puri P, Sanyal AJ (2011) The multidimensional burden of cirrhosis and hepatic encephalopathy on patients and caregivers. Am J Gastroenterol 106(9):1646-1653. doi: 10.1038/ajg.2011.157

Baumgartner RN (2000) Body composition in healthy aging. Ann N Y Acad Sci 904:437-448

Baumgartner RN, Koehler KM, Gallagher D, Romero L, Heymsfield SB, Ross RR, Garry PJ, Lindeman RD (1998) Epidemiology of sarcopenia among the elderly in New Mexico. Am J Epidemiol 147:755-763. doi:10.1093/oxfordjournals.aje.a009520
Bémeur C, Desjardins P, Butterworth RF (2010) Role of nutrition in the management of hepatic encephalopathy in end-stage liver failure. J Nutr Metab 2010:489823. doi:10.1155/2010/489823

Berzigotti A, Garcia-Tsao G, Bosch J, Grace ND, BurrougHGS AK, Morillas R, Escorsell A, Garcia-Pagan JC, Patch D, Matloff DS, Groszmann RJ, Portal Hypertension Collaborative Group (2011) Obesity is an independent risk factor for clinical decompensation in patients with cirrhosis. Hepatology 54:555-561. doi:10.1002/ hep. 24418

Bianchi G, Giovagnoli M, Sasdelli AS, Marchesini G (2012) Hepatic encephalopathy and health-related quality of life. Clin Liver Dis 16(1):159-170. doi:10.1016/j.cld.2011.12.003

Bielemann RM, Horta BL, Orlandi SP, Barbosa-Silva TG, Gonzalez MC, Assunção MC, Gigante DP (2015) Is adductor pollicis muscle thickness a good predictor of lean mass in adults? Clin Nutr. doi:10.1016/ j.clnu.2015.07.022

Blackburn GL, Harvey KB (1982) Nutritional assessment as a routine in clinical medicine. Postgrad Med 71:46-63

Blei AT (2000) Diagnosis and treatment of hepatic encephalopathy. Baillieres Best Pract Res Clin Gastroenterol 14(6):959-974

Blei AT, Córdoba J (2001) Hepatic encephalopathy. Practice parameters Committee of the American College of gastroenterology. Am J Gastroenterol 96(7):1968-1976

Butterworth RF (2002) Pathophysiology of hepatic encephalopathy: a new look at ammonia. Metab Brain Dis 17(4):221-227

Callaway CW, Chumlea WC, Bouchard C, Himes JH, Lohman TG, Martin AD, et al. (1988) Circumferences. In: Lohman TG, Roche AF, Martorell R (eds) Anthropometric standardizing reference manual. Human Kinetics, Champaign, pp. 39-54

Caporossi FS, Caporossi C, Borges Dock-Nascimento D, de AguilarNascimento JE (2012) Measurement of the thickness of the adductor pollicis muscle as a predictor of outcome in critically ill patients. Nutr Hosp 27(2):490-495. doi:10.1590/S0212-16112012000200021

Carvalho L, Parise ER (2006) Evaluation of nutritional status of nonhospitalized patients with liver cirrhosis. Arq Gastroenterol 43(4):269274

Chadalavada R, Biyyani RSS, Maxwell J, Muleen K (2010) Nutrition in hepatic encephalopathy. Nutr Clin Pract 5:257-264. doi:10.1177/ 0884533610368712

Cheung K, Lee S, Raman M (2012) Prevalence and mechanisms of malnutrition in patients with advanced liver disease, and nutrition management strategies. Clin Gastroenterol Hepatol 10:117-125. doi:10. 1016/j.cgh.2011.08.016

de Oliveira CM, Kubrusly M, Mota RS, Choukroun G, Neto JB, da Silva CA (2012) Adductor pollicis muscle thickness: a promising anthropometric parameter for patients with chronic renal failure. J Ren Nutr 22(3):307-316. doi:10.1053/j.jrn.2011.07.006

Figueiredo FA, Dickson ER, Pasha TM, Porayko MK, Therneau TM, Malinchoc M, et al. (2000) Utility of standard nutritional parameters in detecting body cell mass depletion in patients with end-stage liver disease. Liver Transpl 6:575-581

Harrison GG, Buskirk EK, Carter JEL, Ohmston JFE, Lohman TG, Pollock ML, et al. (1988) Skinfold thicknesses and measurements technique. In: Lohman TG, Roche AF, Martorell R (eds) Anthropometric standardizing reference manual. Human Kinetics, Champaign, pp. $55-80$

Hayashi F, Matsumoto Y, Momoki C, Yuikawa M, Okada G, Hamakawa E, et al. (2013) Physical inactivity and insufficient dietary intake are associated with the frequency of sarcopenia in patients with compensated viral liver cirrhosis. Hepatol Res 43(12):1264-1275

Huisman EJ, Trip EJ, Siersema PD, van Hoek B, van Erpecum KJ (2011) Protein energy malnutrition predicts complications in liver cirrhosis. Eur J Gastroenterol Hepatol 23:982-989. doi:10.1097/MEG. 0b013e32834aa4bb

Iwasa M, Hara N, Terasaka E, Hattori A, Ishidome M, Mifuji-Moroka R, Miyachi H, Sugimoto R, Tanaka H, Fujita N, Kobayashi Y, Iwata K, 
Takei Y (2014) Evaluation and prognosis of sarcopenia using impedance analysis in patients with liver cirrhosis. Hepatol Res 44(10): E316-E317. doi:10.1111/hepr.12217

James R (1989) Nutritional support in alcoholic liver disease: a review. J Hum Nutr 2315-23

Jeejeebhoy KN (2012) Malnutrition, fatigue, frailty, vulnerability, sarcopenia and cachexia: overlap of clinical features. Curr Opin Clin Nutr Metab Care 15:213-219. doi:10.1097/MCO.0b013e328352694f

Kalaitzakis E, Olsson R, Henfridsson P, Hugosson I, Bengtsson M, Jalan $\mathrm{R}$, Björnsson E (2007) Malnutrition and diabetes mellitus are related to hepatic encephalopathy in patients with liver cirrhosis. Liver Int 27:1194-1201. doi:10.1111/j.1478-3231.2007.01562.x

Lameu EB, Gerude MF, Campos AC, Luiz RR (2004) The thickness of the adductor pollicis muscle reflects the muscle compartment and may be used as a new anthropometric parameter for nutritional assessment. Curr Opin Clin Nutr Metab Care 7(3):293-301

Leong Shu-Fen C, Ong V, Kowitlawakul Y, Ling TA, Mukhopadhyay A, Henry J (2015) The adductor pollicis muscle: a poor predictor of clinical outcome in ICU patients. Asia Pac J Clin Nutr 24(4):605609. doi:10.6133/apjen.2015.24.4.22

McCullough AJ, Mullen KD, Kalhan SC (1991) Measurements of total body and extracellular water in cirrhotic patients with and without ascites. Hepatology 14:1102-1111. doi:10.1002/hep.1840140626

Merli M, Giusto M, Lucidi C, Giannelli V, Pentassuglio I, Di Gregorio V, Lattanzi B, Riggio O (2013) Muscle depletion increases the risk of overt and minimal hepatic encephalopathy: results of a prospective study. Metab Brain Dis 28(2):281-284. doi:10.1007/s11011-0129365-z

Montano-Loza AJ, Meza-Junco J, Prado CM, Lieffers JR, Baracos VE, Bain VG, Sawyer MB (2012) Muscle wasting is associated with mortality in patients with cirrhosis. Clin Gastroenterol Hepatol 10: 166-173. doi:10.1016/j.cgh.2011.08.028

Morano GN, Seibyl JP (2003) Technical overview of brain SPECT imaging: improving acquisition and processing of data. J Nucl Med Technol 31:191-195

Peng S, Plank LD, McCall JL, Gillanders LK, McIlroy K, Gane EJ (2007) Body composition, muscle function, and energy expenditure in patients with liver cirrhosis: a comprehensive study. Am J Clin Nutr 85:1257-1266

Periyalwar P, Dasarathy S (2012) Malnutrition in cirrhosis: contribution and consequences of sarcopenia on metabolic and clinical responses. Clin Liver Dis 16:95-131. doi:10.1016/j.cld.2011.12.009

Plauth M, Cabré E, Riggio O, Assis-Camilo M, Pirlich M, Kondrup J (2006) ESPEN guidelines on enteral nutrition: liver disease. Clin Nutr 25:285-294. doi:10.1016/j.clnu.2006.01.018
Poziomyck AK, Weston AC, Lameu EB, Cassol OS, Coelho LJ, Moreira LF (2012) Preoperative nutritional assessment and prognosis in patients with foregut tumors. Nutr Cancer 64(8):1174-1181. doi:10. 1080/01635581.2012.721157

Prado CMM, Wells JCK, Smith SR, Stephan BCM, Siervo M (2012) Sarcopenic obesity: a critical appraisal of the current evidence. Clin Nutr 31:583-601

Riggio O, Andreoli A, Diana F, Fiore P, Meddi P, Lionetti R, Montagnese F, Merli M, Capocaccia L, De Lorenzo A (1997) Whole body and regional body composition analysis by dual-energy X-ray absorptiometry in cirrhotic patients. Eur J Clin Nutr 51:810-814

Romeiro FG, Augusti L (2015) Nutritional assessment in cirrhotic patients with hepatic encephalopathy. World J Hepatol 7(30):2940 2954. doi:10.4254/wjh.v7.i30.2940

Romero-Gómez M, Montagnese S, Jalan R (2015) Hepatic encephalopathy in patients with acute decompensation of cirrhosis and acuteon-chronic liver failure. J Hepatol 62:437-447. doi:10.1016/j.jhep. 2014.09.005

Stewart CA, Malinchoc M, Kim WR, Kamath PS (2007) Hepatic encephalopathy as a predictor of survival in patients with end-stage liver disease. Liver Transpl 13:1366-1371. doi:10.1002/1t.21129

Sunil HV, Mittal BR, Kurmi R, Chawla YK, Dhiman RK (2012) Brain perfusion single photon emission computed tomography abnormalities in patients with minimal hepatic encephalopathy. J Clin Exp Hepatol 2(2):116-121

Tapper EB, Jiang ZG, Patwardhan VR (2015) Refining the Ammonia Hypothesis: A Physiology-Driven Approach to the Treatment of Hepatic Encephalopathy. Mayo Clin Proc 90(5):646-658. doi:10. 1016/j.mayocp.2015.03.003

Vilstrup H, Amodio P, Bajaj J, Cordoba J, Ferenci P, Mullen KD, Weissenborn K, Wong P (2014a) Hepatic encephalopathy in chronic liver disease: 2014 Practice Guideline by the American Association for the Study of Liver Diseases and the European Association for the Study of the Liver. Hepatology 60:715-735. doi:10.1002/hep. 27210

Vilstrup H, Amodio P, Bajaj J, Cordoba J, Ferenci P, Mullen KD, Weissenborn K, Wong P (2014b) Hepatic encephalopathy in chronic liver disease: 2014 practice guideline by the European Association for the Study of the liver and the American Association for the Study of Liver Diseases. American Association for the Study of Liver Diseases; European Association for the Study of the liver. J Hepatol 61(3):642-659. doi:10.1016/j.jhep.2014.05.042

Waters DL, Qualls CR, Dorin RI, Veldhuis JD, Baumgartner RN (2008) Altered growth hormone, cortisol, and leptin secretion in healthy elderly persons with sarcopenia and mixed body composition phenotypes. J Gerontol A Biol Sci Med Sci 63(5):536e41 